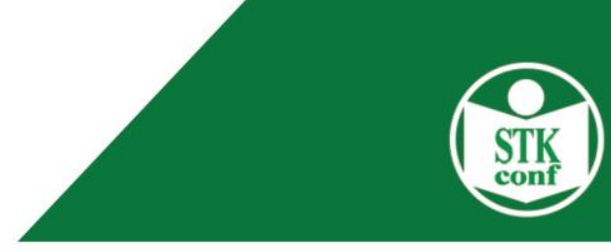

26-28 February, 2021

Amsterdam, Netherlands

\title{
Knowledge Representation in Business Process Automation Systems
}

\author{
Petrika Manika ${ }^{1}$, Endrit Xhina ${ }^{2}$, Ana Ktona ${ }^{3}$ \\ ${ }^{1,2,3}$ University of Tirana, Faculty of Natural Sciences, Department of Informatics, Albania
}

\begin{abstract}
.
Knowledge representation is one of the most familiar concept fields of artificial intelligence. A large number of studies have been done for representation varieties and also for the structure of a knowledge representation. The properties that a data set should have to be defined as knowledge representation are also very important. In knowledge representation, an ontology is used to describe a particular approach to the representation of concepts and their relationships. Extracting knowledge representation from existing information systems is not a simple or standard process that can be followed by everyone. A lot of knowledge can be generated from business process automation systems. The purpose of this paper is to analyse the knowledge representation that can be extracted from the huge amount of data that a business process automation system can have. Smart Processes is a business process automation web system that is used in this paper as a case study to generate knowledge.
\end{abstract}

Keywords: artificial intelligence, knowledge representation, ontologies, business process automation, smart processes

\section{Introduction}

Artificial Intelligence (AI) is a branch of computer sciences that studies the construction of intelligent components that can think and behave like humans. Some examples of AI in action are learning, speech recognition, problem-solving and making decisions. AI works on two main aspects when building a machine, to make machine behaviours the same as human behaviours and simulating the human brain to learn new things, to interpret different situations and to solve problems (Bozzato et al., 2018). If scientists work hard on these two aspects then the simulation of human intelligence can be achieved. One of the characteristic of artificial intelligence is to take actions that have the best chance of achieving a specific goal, but for a machine to have the ability to reason and know how to act in a given situation it must have a lot of knowledge.

Knowledge representation is the most familiar concept in AI. The main question is what is a knowledge representation. This question is rarely been answered directly. Numerous studies have been done recently and many articles have been written which focus on the variety of representation, the properties that a representation should have and the appropriate structures of knowledge representation. In artificial intelligence, knowledge representation is the study of how the beliefs, intentions, and value judgments of an intelligent agent can be expressed in a transparent, symbolic notation suitable for automated reasoning (Bozzato et al., 2018). Some 


\section{$5^{\text {th }}$ International Conference On Applied Research In SCIENCE, TECHNOLOGY \& KNOWLEDGE}

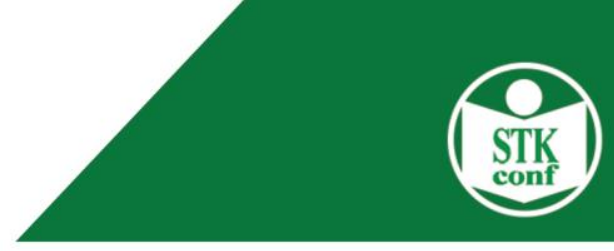

26-28 February, 2021

Amsterdam, Netherlands

of the architectures that have been used for knowledge representation are formal logics, firstorder logic, semantic networks and frame. The properties that a data set should have to be defined as knowledge representation are also very important. The source from which the knowledge will come and the way or the structure they will be represented have always been difficult. Let's start with an analysis for the source from which we can build knowledge (Bonatti et al.,2015).

Knowledge representation will be extracted from massive data. Data can be collected from different information systems as well as from various documents that can be found on the internet. Software generates and processes a large amount of data every day, however data obtained from information systems and the World Wide Web cannot be used directly as a representation of knowledge. They must first be processed and then structured in an acceptable manner following certain rules. Data can be generated in the form of raw data which are easily produced by information systems. But then the transformation of this data into knowledge representation requires the use of different techniques that include data analysis, data mining, etc (Berčič and Vintar, 2004). The use of machine learning techniques for the automatic construction of knowledge bases and knowledge representations is difficult, but has achieved some degree of success. A new and best approach to build knowledge representation is that information systems themselves, or the World Wide Web itself produce a structured content that may be appropriate as a knowledge representation. Both methods are described in Fig.1.

Figure 1: Generating knowledge representation
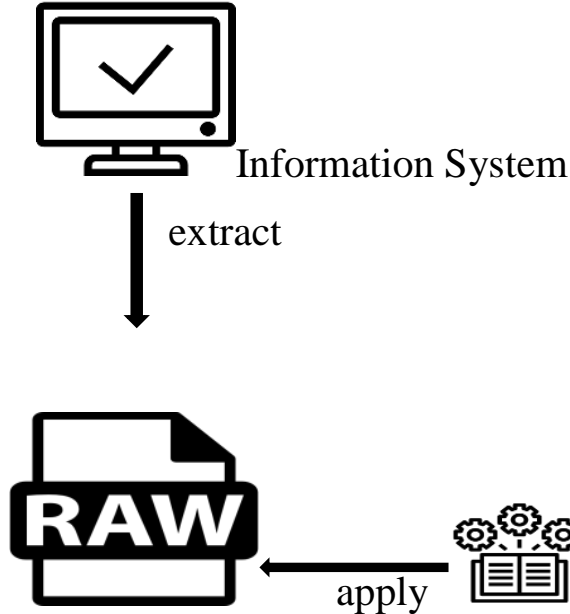

Raw data

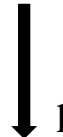

produce apply

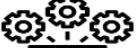

\section{,}
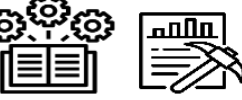

ML and DM
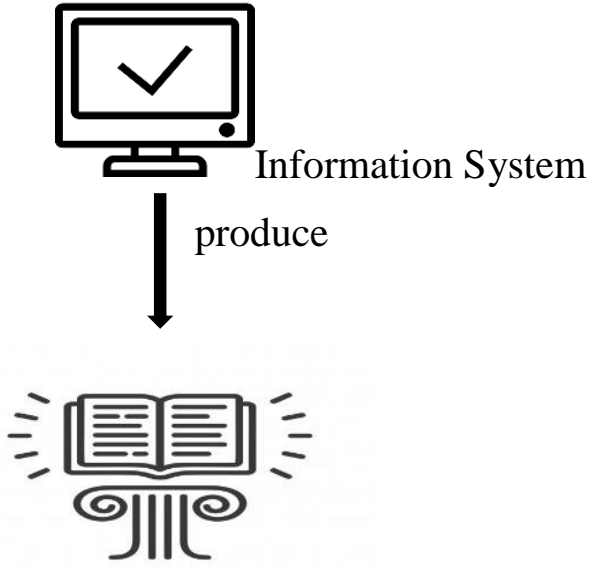

Knowledge Representation

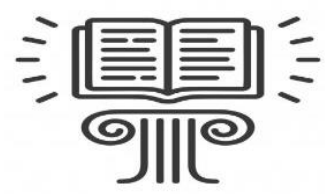

Knowledge Representation 


\section{$5^{\text {th }}$ International Conference On Applied Research In SCIENCE, TECHNOLOGY \& KNOWLEDGE}

26-28 February, 2021

Amsterdam, Netherlands

The first approach is more clear than the second. Extracting raw data is easy, but then the application of machine learning techniques is a procedure that requires specialized human intervention and time. While the second approach is more fast and more secure but is more difficult to realize. This paper will introduce ontologies as a structure to represent knowledge. Ontologies are part of the semantic web. Also this paper will introduce the possibility of applying the second approach to produce knowledge representation from a business process automation system that manages big data. The system that has been studied is Smart Processes.

\section{Knowledge representation techniques}

When you think of knowledge representation you maybe think about facts, procedures, meaning, etc. Choosing the wrong representation could lead to fail. There are four general knowledge representation types: logical representations, semantic networks, production rules and frames (Randall et al., 2002). Each of the general techniques for building knowledge representation has its advantages and disadvantages that will be analysed below.

\section{Figure 2: Knowledge representation general techniques}

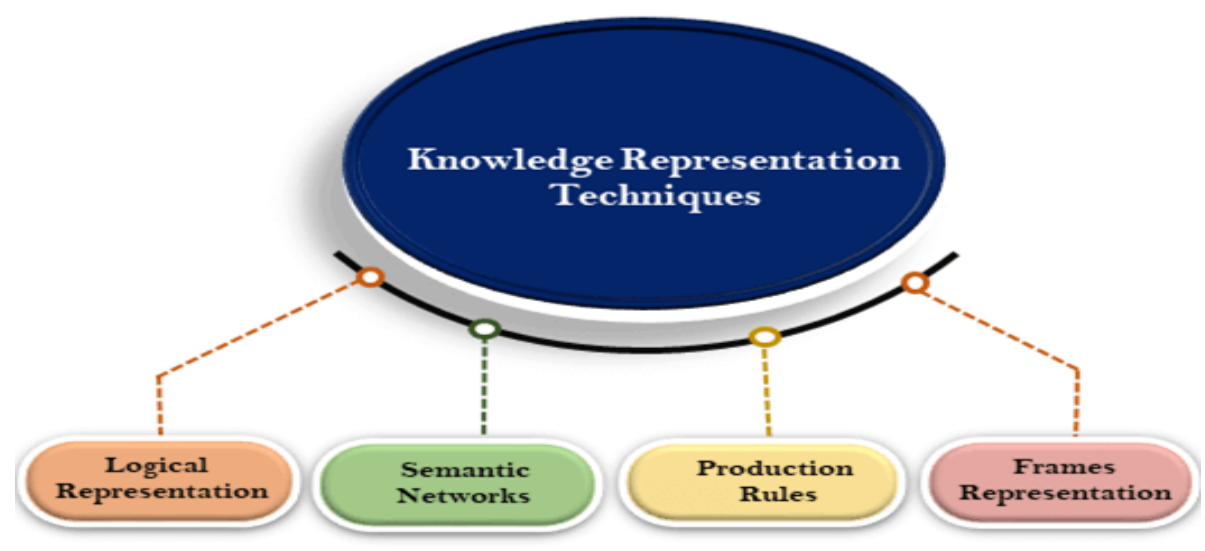

\subsection{Logical representation}

Logical representation is a language that have rules to be followed. These rules deals with propositions and the language has no ambiguity in representation. Using logical representation we gain conclusions as output based on some conditions. The two most important parts of this technique are the syntax and semantics. The syntax is a set of rules that decide the way we can construct legal sentences and how to write symbols in knowledge representation. We can assign a meaning to each sentence using semantic, thanks to the rules by which we are able to interpret the sentence in the logic. The two main categories of logical representation are propositional logics and predicate logics. Using logical representation enables us to do logical reasoning, but is challenging to work with due to some restrictions and also is not very natural (Bonatti et al.,2015).

\subsection{Semantic network representation}

If we want to represent knowledge in the form of graphical networks, we can use semantic networks. In this type of representation two are the main parts: nodes that represent objects and 


\section{$5^{\text {th }}$ International Conference On Applied Research In SCIENCE, TECHNOLOGY \& KNOWLEDGE}

26-28 February, 2021

Amsterdam, Netherlands

arcs that represent the relationships between those objects. The object can be categorized in different forms. Some advantages of semantic networks are ease of understanding, the possibility of expansion, the natural way and the transparent manner of meaning presentation. Below is an SNR example for the following statements (Randall et al., 2002):

- Order is a process.

- The process is owned by the administrator.

- Administrator is a user of the process.

- Bill is a user that can make orders.

Figure 3: Semantic network representation

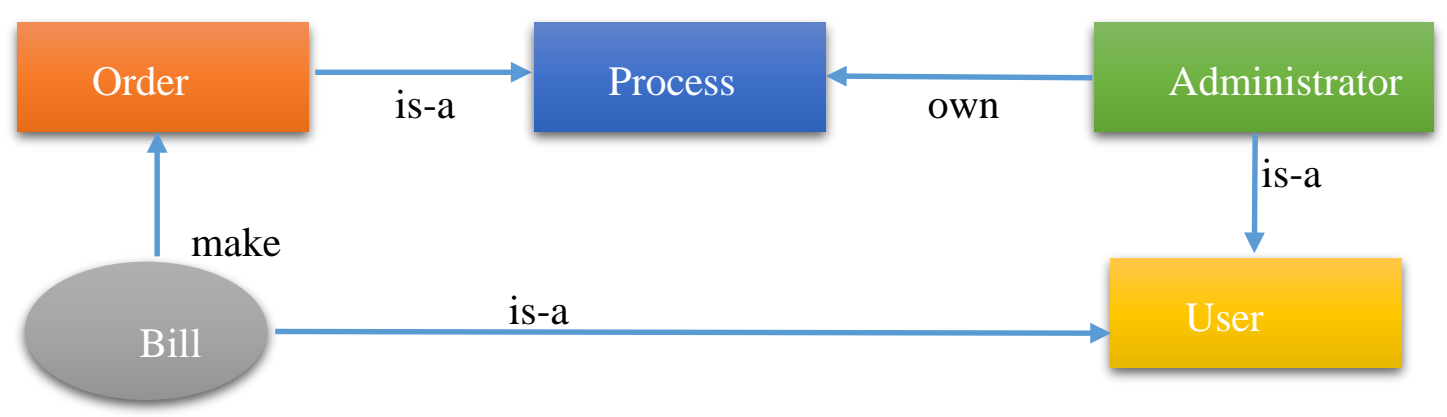

Some disadvantages of this technique are the large computational time in runtime due to graph algorithms, no equivalent quantifier as for all, for some, etc.

\subsection{Production rule}

This type of technique consists in pairs of condition and action. The action is executed if the condition is evaluated to true. This technique has the set of production rules, the working memory and the recognize-act-cycle. If the condition is evaluated to true, production rule fires and the action is executed. The action has encapsulated the problem-solving steps and the condition part specify which rule may be applied to a problem. The working memory contains the description of the current state of problems-solving and rule can write knowledge to the working memory. This knowledge match and may fire other rules (Bruijn et al., 2010). Example:

- IF (value of field days is less than or equal to 31 AND the state is waiting) THEN action (execute send to destroy step).

- IF (send to secondary branch button is clicked) THAN action (send email to owner).

We can easy modify a rule in production rules and rules are expressed in a natural way. Production rules cannot develop any learning capabilities.

\subsection{Frame}

A frame is an AI data structure that describes an entity. It is a collection of attributes and values, like a record. Frames divides knowledge into substructures by representing stereotypes situations. Attributes are called slots. Slots can be of any type and size and have names and values which are called facets. Using facets we can put constraints on the frames. A frame can have any number of slots, a slot can have any number of facets and a facet can have any number of values (Bruijn et al., 2010). Frames are derived from semantic networks. Frames are used in natural language processing. Tab.1 shows an example of frame for a process. 


\section{$5^{\text {th }}$ International Conference On Applied Research In SCIENCE, TECHNOLOGY \& KNOWLEDGE}

26-28 February, 2021

Amsterdam, Netherlands

Table 1:Frame

\begin{tabular}{|l|l|}
\hline Slots & Filters \\
\hline Name & Orders \\
\hline Date of creation & $21-12-2020$ \\
\hline Maximal number of open cases & 100 \\
\hline Type & General \\
\hline Owner & Administrator \\
\hline
\end{tabular}

Frame representation makes programming easier because is flexible. We can add slots for new attribute in an easy way and is easy to understand and visualize. The main disadvantage of frame representations is that the inference mechanism is not easily processed. Also frame representations have a much generalized way.

\section{Semantic web}

The term Semantic Web refers to the World Wide Web Consortium vision for a data-driven Web (World Wide Web Consortium 2020). The technologies of semantic can build dictionaries that model knowledge of various fields, store intermediate data, and write rules to manage data. The semantics infrastructure and architecture of the web provides the right support for knowledge representation and concept definition. As shown in Fig.4, (Berners Lee, 2006), an important part of semantic web are ontologies, which allow the presentation of classes that describe concepts, contain attributes for each class, and the relationships that may exist between them.

Figure 4: Semantic web

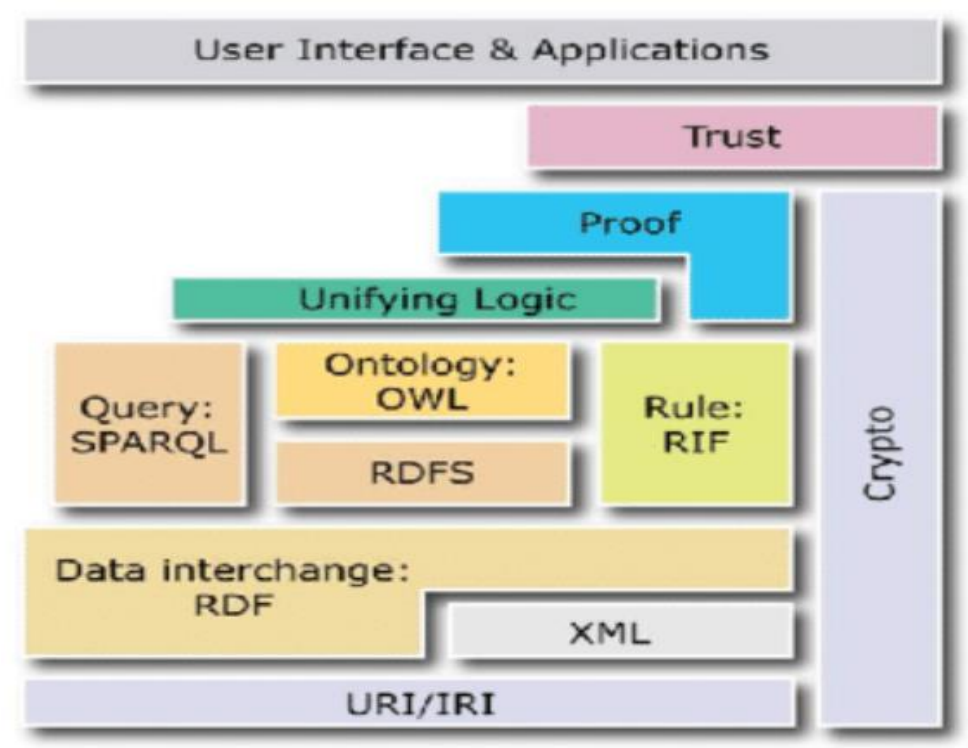




\section{$5^{\text {th }}$ International Conference On Applied Research In SCIENCE, TECHNOLOGY \& KNOWLEDGE}

26-28 February, 2021

Amsterdam, Netherlands

\subsection{Ontologies}

The term ontology comes from philosophy, as a subfield of philosophy that studies the nature of existence. In computer science, ontology is a formal representation of knowledge from a set of concepts within a domain and the relationships between these concepts (Antoniou et al.2012). In the last decade, the concept of ontology has spread to areas such as intelligent information integration, information extraction, e-commerce and knowledge management (Theocharis \& Tsihrintzis, 2012). Ontologies are used to represent objects in a domain and to describe their attributes. Ontologies are used to describe the connections that objects have with each other.

Ontologies can be of different types:

1. High-level ontologies would describe very general concepts such as space, time, matter, object, event, and action, which are independent of a specific problem or field.

2. Domain ontologies describe the vocabulary related to a general field (medicine, transport).

3. Application ontologies describe concepts that depend on both the field and the tasks. These concepts would correspond to the roles played by domain entities while performing a particular activity.

Representation of facts can be done in different ways. One of the challenges of semantic web is for computers to be able to understand the content of information. Information can be provided in XML (eXtensible Markup Language) format. The representation of information in XML stay at the syntactic level because it does not give meaning to information. Relationships between objects and attributes cannot be defined if we use XML. RDF(Resource Description Framework) is a semantic web language (World Wide Web Consortium 2020). Resource stands for source, in the field of process automation we could have resources: process, actor, workflow, document, etc. Description stands for the description of relationships between sources. The framework is for combining web protocols such as XML, HTTP, etc. Data can be structured in this model through what are called triples or three-lines. A triples would be so named because it would consist of source, predicate and object / value, so three elements. RDF is sufficient for simple ontologies but for modeling complex knowledge, languages based on formal logic are needed. OWL (Ontology Web Language) is a declarative language that describes the state of objects logically. Two versions according to the W3C are OWL since 2004 and OWL 2 since 2009 (World Wide Web Consortium 2020). Ontologies can be expressed in OWL language and then certain tools named logic tools can be used to deduce further information. The OWL language contains information about classes, class hierarchies, attributes, attribute hierarchies, and so-called instances or individuals.

\section{Business process automation systems}

The development of information systems that model business processes and automate processes has received great attention in the last decade (Watson and Holmes, 2009). Industry and businesses carry out different processes every day. Work processes involve different actors, have their own characteristics, workflow, constraints, duration, business rules etc (Odawa et al., 2014). For a process to be built correctly and executed properly requires a lot of knowledge and in-depth analysis. Nowadays many businesses don't have process excellence and workflow automation. Process excellence is a very important point in the success of a business. Business process automation is the use of technology to execute recurring tasks or processes in a business 


\section{$5^{\text {th }}$ International Conference On Applied Research In SCIENCE, TECHNOLOGY \& KNOWLEDGE}

26-28 February, 2021

Amsterdam, Netherlands

where manual effort can be replaced. It is done to minimize costs, increase efficiency, and streamline processes (Bridgeland and Zahavi, 2009). Without automation, the entire process can become quite chaotic and result in endless paperwork, missing out on some tasks, employee dissatisfaction and low productivity. Process automation lead to digital transformation, more clarity, standardize operations, increase satisfaction and efficiency. It accelerates how work gets done by routing information to the right person at the right time through user-defined rules and actions.

\subsection{Smart Processes}

Smart Processes is a highly flexible web based software solution for business process automation (Smart Processes 2020). It could accommodate different scenarios, serving multiple types of businesses and institutions. Smart Processes is an ideal solution to implement a standard process to many scenarios from several application fields in different industries, including banking, educations, government, insurance, etc. Fig.5 shows the main modules of this software.

Figure 5: Smart Processes Modules

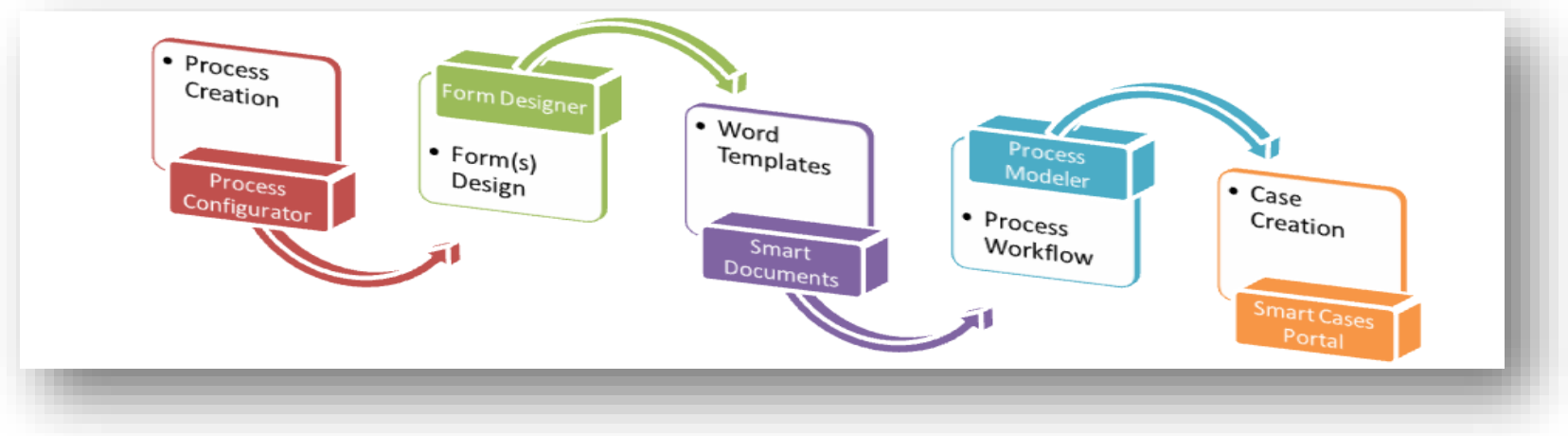

Smart Processes is equipped with the necessary tools to design and execute processes:

- Process Configurator - creates and configures processes in terms of capacity, availability, events and access rights.

- Form Designer - creates data entry forms which serve as a data storage for the cases of the process. Forms are also the place where the users interact with the Smart Process management engine.

- Smart Documents - provide the mean to generate pre-formatted documents with data of the cases.

- Process Modeler - is used to model the workflow of the process. It creates the statuses and steps upon which cases of the process will flow. It allows also creating business rules for routing cases through the defined process statuses based on data entered in the cases and external events.

- Business Rules Designer - provides the functionality to create complex formulas and expressions upon the data of the process cases.

- Cases Portal - provides the environment where users of the application create and execute cases of the process.

- User Roles and Access Rights(s) - provides the interface for adding and removing users from the process access list. 


\section{$5^{\text {th }}$ International Conference On Applied Research In SCIENCE, TECHNOLOGY \& KNOWLEDGE}

26-28 February, 2021

Amsterdam, Netherlands

\subsection{Knowledge representation in Smart Processes}

Smart Processes is a process automation software through which business processes with different characteristics can be built (Smart Processes 2020). The process configuration goes through several steps described by the figure below:

Figure 6: Process configuration

\section{Configurator / Đ Form Designer / @Modeler / छ Events / ̊ Users, Roles \& Permissions}

Configurator - the general data of the process are defined as name, description, type, category, start date, end date, cases capabilities, etc.

Form Designer - creates data entry forms with fields that serve as a data storage for the cases of the process. Fields can be of different types likes textfield, radio button, select list, date field, etc.

Modeler - creates the workflow of the process. Workflow determines the start status, intermediate statuses and the end status that follow all the cases of the process. Cases execute steps and one step has two statuses: the initial status and the final status.

Events - configure the events that can occur at the cases of the process and actions that are executed for each event.

User Roles \& Permissions - define all access rules that apply to process cases based on users, forms and workflow.

The extraction of knowledge representation directly from smart processes can be achieved based on the relationships that exist between the domain objects. Knowledge can be extracted from process metadata, workflow, forms, events or access rules. The knowledge that can be generated from the workflow of a process and from the fields of forms is analyzed below.

Once a process is created in smart processes, based on the access rules that are configured for the process, eligible users can create process instances which are called cases. A case is an instance of the process for which the values of the form fields are set, steps are executed to go from one status to another based on the workflow configured for the process, events are heard and actions are executed based on the event. An example of a process might be Orders with the following configuration:

Table 2:Orders process metadata

\begin{tabular}{|l|l|}
\hline Name & Orders \\
\hline Description & Process for managing orders \\
\hline Type & Process \\
\hline Allow Case Creation & Yes \\
\hline Start Date & $21 / 11 / 2020$ \\
\hline End Date & $21 / 11 / 2021$ \\
\hline Sharing Level & Office Share \\
\hline Cases Total Capacity & 20000 \\
\hline
\end{tabular}




\section{$5^{\text {th }}$ International Conference On Applied Research In SCIENCE, TECHNOLOGY \& KNOWLEDGE}

26-28 February, 2021

Amsterdam, Netherlands

Figure 6: Order process workflow

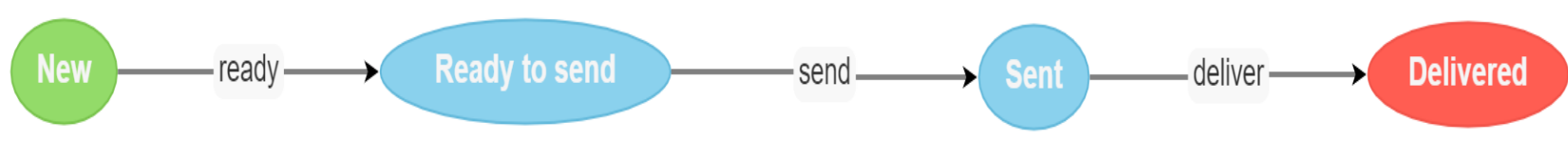

Start status: New

Intermediate status: Ready to send, Sent

End status: Delivered

Steps: ready, send, deliver

Figure 7: Process forms and fields

\begin{tabular}{|l|l|}
\hline Order Information & Order Date \\
\hline Order number & $\mathrm{mm} / \mathrm{dd} / \mathrm{yyyy}$ \\
\hline Order Description & Items \\
\hline & Clothes \\
\hline Client full name & Electronics \\
\hline
\end{tabular}

Order send information

Send date
mm/dd/yyyy

Sender name

Description

Delivered information

Delivered date

Description

$\mathrm{mm} / \mathrm{dd} /$ yyyy

$\square$

Delivered

Knowledge can be represented using ontologies (Osterwalder et al., 2004). The workflow ontology is as follows: 


\section{$5^{\text {th }}$ International Conference On Applied Research In SCIENCE, TECHNOLOGY \& KNOWLEDGE}

Figure 8: Process workflow ontology

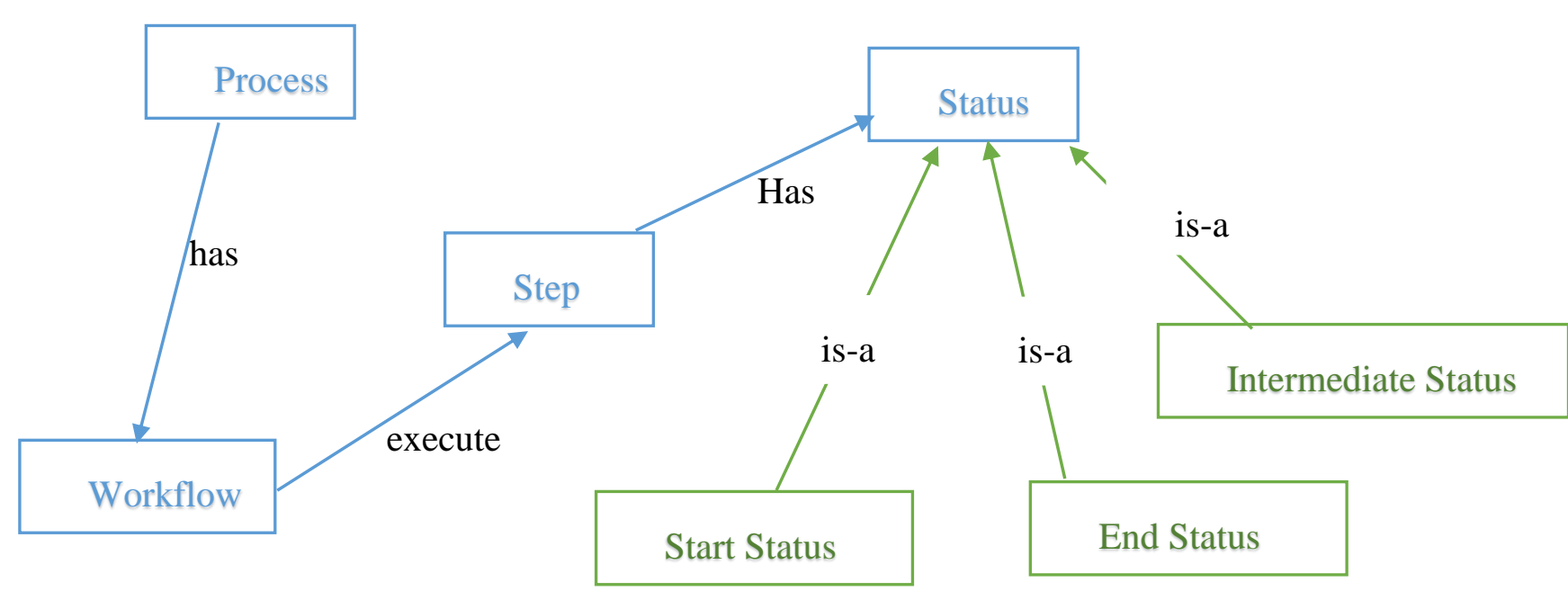

Since the ontology must be filled with individuals, these are taken from the process configuration data whenever a new process is configured. The smart processes database diagram for the workflow includes the tables below:

Figure 9: Database diagram for workflow

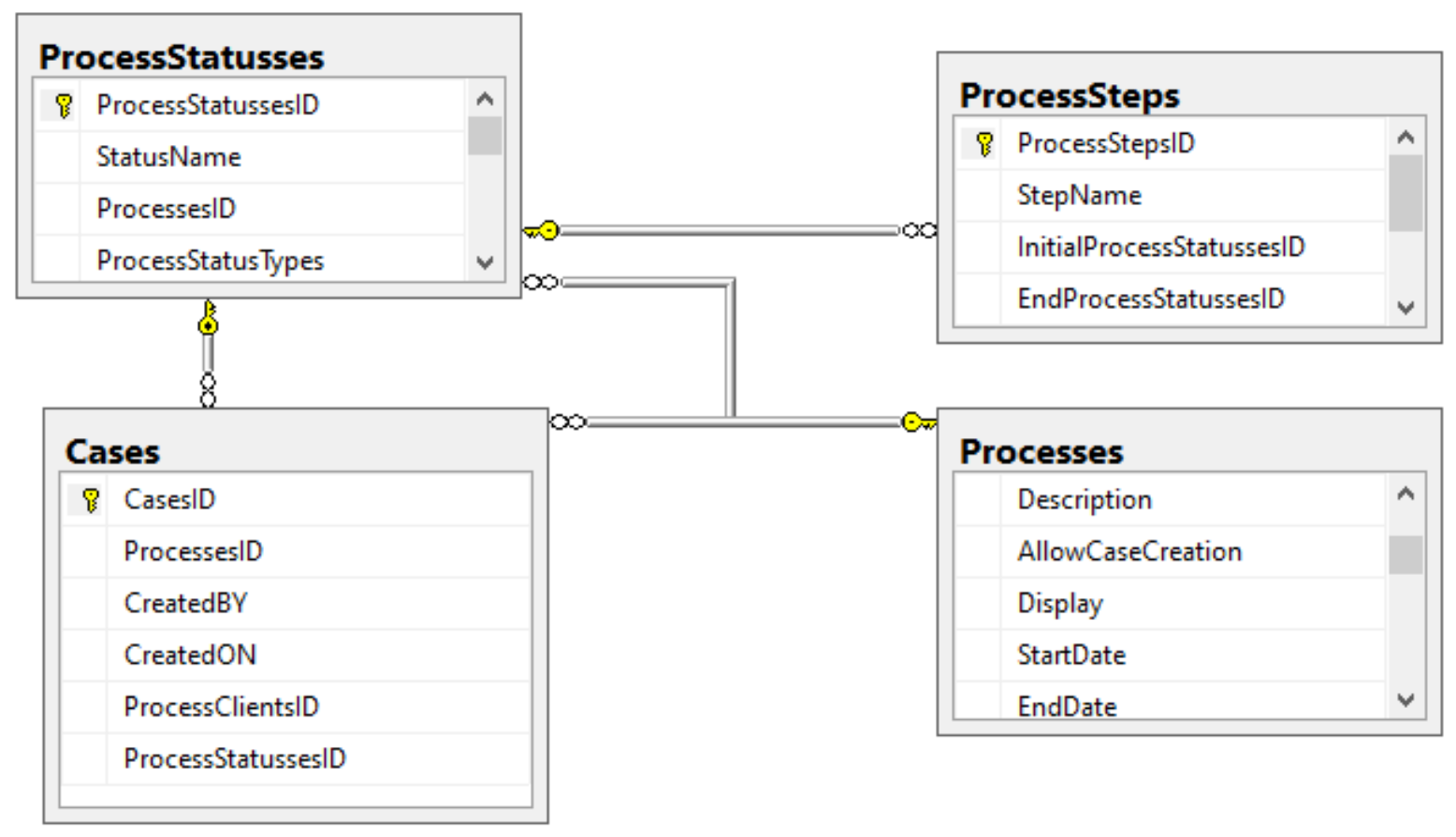

Another knowledge representation can be extracted from process fields. The fields ontology is as follows: 


\section{$5^{\text {th }}$ International Conference On Applied Research In SCIENCE, TECHNOLOGY \& KNOWLEDGE}

26-28 February, 2021 Amsterdam, Netherlands

Figure 10: Process fields ontology

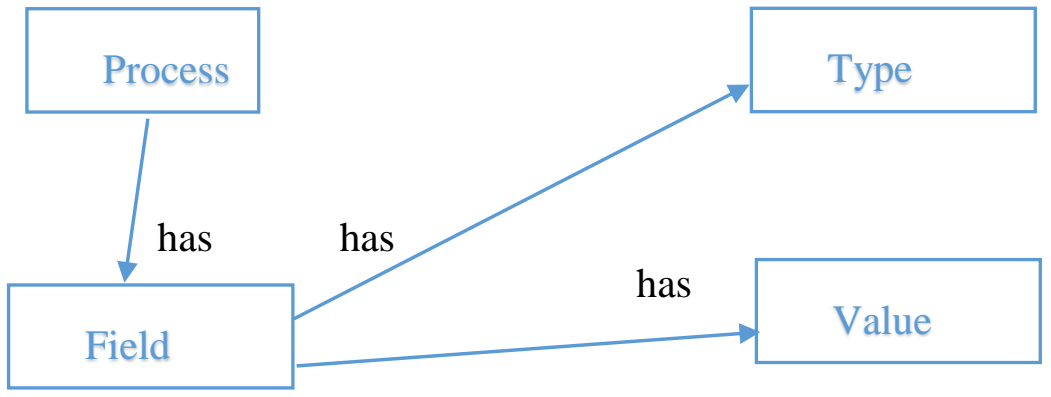

Individuals of ontology are taken from cases of process. The smart processes database diagram for cases and fields includes the tables below:

Figure 11: Database diagram for cases and fields

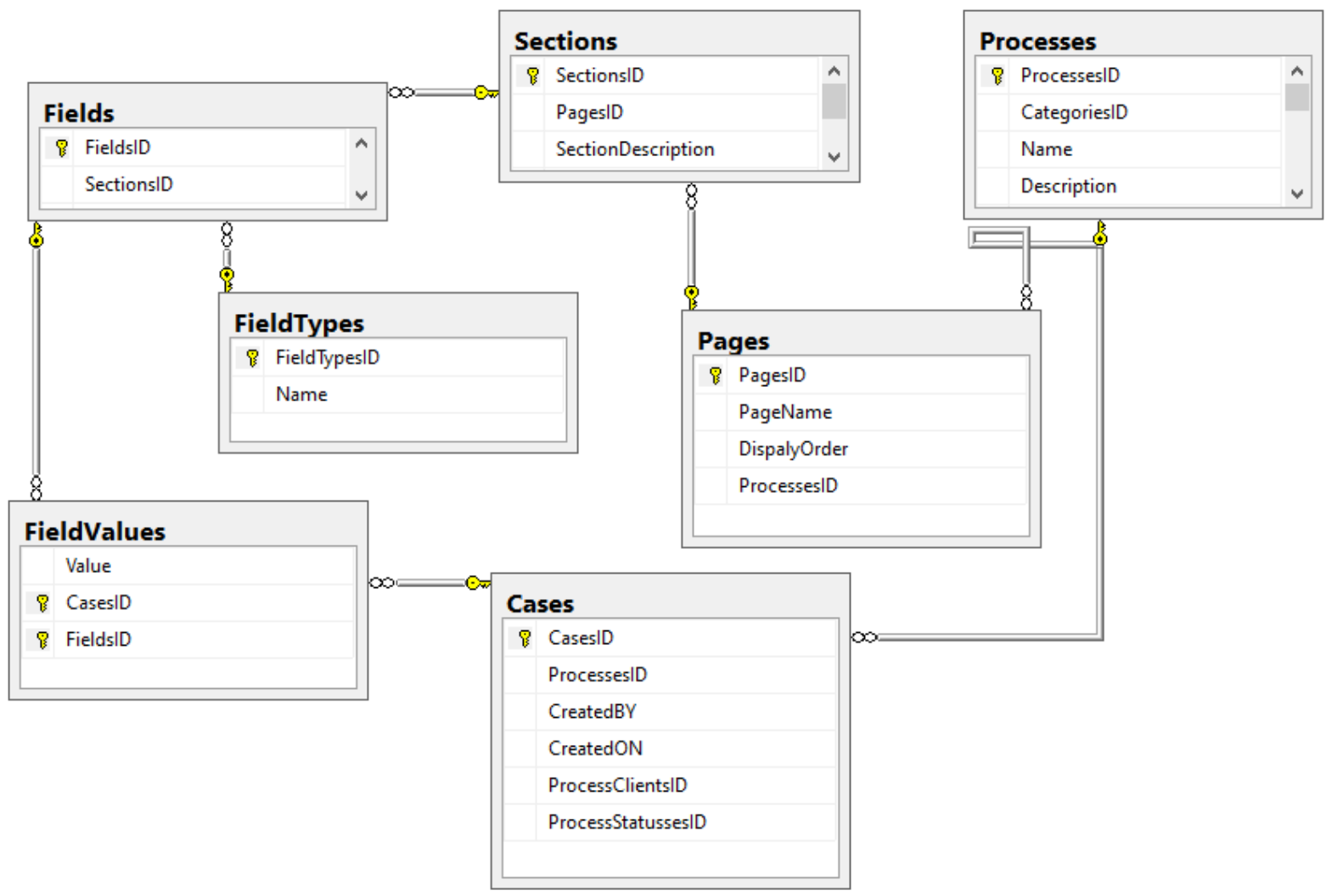

Based on the ontology in Fig.8 and connections between objects a procedure can be built that produces an OWL file for each workflow process. Regarding the ontology for cases and fields, a procedure can be built that produces an OWL file for each case of the process. Using this methodology we can obtain knowledge representation directly from the data of the business 


\section{$5^{\text {th }}$ International Conference On Applied Research In SCIENCE, TECHNOLOGY \& KNOWLEDGE}

26-28 February, 2021

Amsterdam, Netherlands

process automation system without the need to use different techniques on raw data (Ilayperuma 2007).

\section{Conclusions}

A large number of studies have been done for representation varieties and for the structure of a knowledge representation. There are four general knowledge representation types: logical representations, semantic networks, production rules and frames. In the field of semantic web, an ontology is used to describe a particular approach to the representation of concepts and their inter-relations. Ontologies can be used for representing knowledge. Extracting knowledge representation from existing information systems is not a simple or standard process that can be followed by everyone. Extracting raw data from software is easy, but then the application of machine learning techniques is a procedure that requires specialized human intervention and time. Extracting knowledge representation directly from software is faster and more secure but is more difficult to realize. This paper presented a technique for building ontologies as knowledge representations from the huge amount of data that a business process automation system can have. An example was the Smart Processes system for building knowledge on process workflows and process data.

\section{References}

Theocharis, A. S. and Tsihrintzis, A. G. (2012). "Semantic Web Technologies in eGovernment", World Academy of Science, Engineering and Technology International Journal of Computer and Information Engineering, vol. 6, No.4.

Antoniou, G., Groth, P., Harmelen, V. F. and Hoekstra, R. (2012). A Semantic Web Primer, 3thd ed. London, UK: The MIT Press Cambridge, pp. 10-12.

World Wide Web Consortium. (2020). Web Ontology Language (OWL). Available: https://www.w3.org/OWL/

Berners-Lee, (2006). SW Arch: Same symbols, multiple languages. Available: https://www.w3.org/2006/Talks/0718-aaai-tbl/Overview.html

Berčič B., and Vintar M. (2004). Simple Life-Events Ontology in SU(M)O-KIF. In: Wimmer M.A. Knowledge Management in Electronic Government. KMGov 2004. Lecture Notes in Computer Science, vol 3035. Springer, Berlin, Heidelberg

Bridgeland, D. M, and Zahavi, R. (2009). Business Modeling: A practical Guide to Realizing Business Value. Morgan Kaufmann/Elsevier.

Osterwalder, A., Parent, Ch. and Pigneur, Y. (2004). Setting up an ontology of business models CAiSE'04 Conference, Riga (Latvia).

Ilayperuma Th., Reference Ontology for Business Models Towards Interoperability between Business Modelling Ontologies, Thesis, Computer and Systems Sciences Stockholm University, Sweden 2007.

Odawa, J., Otanga, D. and Wabwoba, F. (2014). Effective Business Process Automation through Process Reengineering: Case of Public a University in Kenya. 


\section{$5^{\text {th }}$ International Conference On Applied Research In SCIENCE, TECHNOLOGY \& KNOWLEDGE}

26-28 February, 2021

Amsterdam, Netherlands

Watson, E., and Holmes, K. (2009). Business Process Automation.

Smart Processes (2020). Available: https://smartprocesses.net/

Bozzato, L., Eiter., Th. and Serafini, L. (2018). Enhancing Context Knowledge Repositories with Justifiable Exceptions. Twenty-Seventh International Joint Conference on Artificial Intelligence. Stockhol, Sweden, Pages 72-126.

Bonatti P., Faella, M., Petrova, I. and Sauro, L. (2015). A new semantics for overriding in description logics. Artificial Intelligence, 222:1-48, 2015

Randall, D., Shrobe, H., and Szolovits, P. (2002). What Is a Knowledge Representation?. AI Magazine. 14.

Bruijn, J., Pearce, D., Polleres, A., and Valverde, A. (2010). A semantical framework for hybrid knowledge bases. Knowledge and Information Systems. 81-104 (2010) 